\title{
Nickel-Catalyzed Defluorinative Coupling of Aliphatic Aldehydes with Trifluoromethyl Alkenes
}

\author{
Jichao Xiao and John Montgomery* \\ Department of Chemistry, University of Michigan, 930 N. University Ave. Ann Arbor, MI 48109-1055, Email: \\ jmontg@umich.edu
}

\begin{abstract}
A simple procedure is reported for the nickel-catalyzed defluorinative alkylation of unactivated aliphatic aldehydes. The process involves the catalytic reductive union of trifluoromethyl alkenes with aldehydes using a nickel complex of a 6,6'-disubstituted bipyridine ligand with zinc metal as the terminal reductant. The protocol is distinguished by its broad substrate scope, mild conditions, and simple catalytic setup. Reaction outcomes are consistent with the intermediacy of an $\alpha$-silyloxy(alkyl)nickel intermediate generated by a low-valent nickel catalyst, silyl electrophile, and the aldehyde substrate.
\end{abstract}

Transition metal-catalyzed reductive coupling reactions that avoid the need for pre-generation of air- and moisture-sensitive organometallic reagents provide an attractive route to highly functionalized synthetic intermediates. ${ }^{[1]}$ Notably, reductive couplings of unsaturated compounds with aldehydes have demonstrated high efficiency for the construction of carbon-carbon bonds in a number of contexts using alkynes, ${ }^{[2]}$ 1,3-dienes, ${ }^{[3]}$ or allenes (Scheme 1A) ${ }^{[4]}$ Couplings of alkenes with aldehydes, however, are more difficult, and methods are often restricted to intramolecular versions ${ }^{[5]}$ highly activated alkenes such as norbornene ${ }^{[6]}$ and methylenecyclopropane ${ }^{[7]}$ or hydroacylations of styrenes ${ }^{[8]}$ Advances using triethylsilyl triflate as promoter enabled considerable improvements in olefin scope to include alkenes with aromatic aldehydes and tertiary aliphatic aldehydes (Scheme 1B). ${ }^{\left[{ }^{9]}\right.}$ More recently, cobalt- and chromium cocatalyzed branch-selective coupling of alkenes with aldehydes through an alkyl chromium intermediate further broadened the scope of substrate combinations tolerated. ${ }^{[10]}$ Additionally, iron-catalyzed transfer hydrogenative coupling of alkenes with aromatic and aliphatic aldehydes $^{[11]}$ and Bronsted acid enabled nickel-catalyzed hydroalkenylation of styrene derivatives with unactivated aldehydes provided further advances. ${ }^{[12]}$ Despite these developments, the majority of current methods for aldehyde-alkene reductive coupling are restricted to aromatic aldehydes, ${ }^{[13]}$ and the direct coupling reaction of abundantly available alkenes with unactivated aliphatic aldehydes still presents challenges in many cases.

An alternative approach for functionalization $\alpha$ to oxygen involves the generation and capture of $\alpha$-oxy radical intermediates, which have been developed as highly useful cross-coupling partners using nickel catalysis. ${ }^{[14]}$ Among these approaches, ketyl radicals offer a versatile platform of reactivity for reversing the traditional electrophilic character of carbonyls and play a pivotal role in numerous bond-forming and bond-breaking processes including ketyl-olefin couplings. ${ }^{[15]}$ The requirement for strong, stoichiometric reductants, however, places practical limits on the synthetic utility of ketyl intermediates generated by classical approaches. ${ }^{[16]}$ Several innovative strategies to generate ketyl radical were recently reported through processes such as concerted proton-coupled electron transfer, ${ }^{[17]}$ Lewis acid-facilitated photocatalytic reduction, ${ }^{[18]}$ redox-neutral photochemical promotion through transient $\alpha$-acetoxy vinyl iodides intermediate, ${ }^{[19]}$ and electrocatalytic reduction. ${ }^{[20]}$

Recent efforts in our laboratory have identified the addition of $\mathrm{Ni}(0)$ to aliphatic aldehydes through the activation by silyl halides as an alternative strategy for promoting reductive cross couplings of aldehydes either involving cyclization of an ynal with alkylation by an alkyl bromide or through direct coupling of the aldehyde with an alkyl bromide. ${ }^{[21]}$ By analogy, we envisioned that trifluoromethylsubstituted alkenes ${ }^{[22]}$ might serves as competent electrophiles in cross couplings with aldehydes under reductive conditions. This outcome would enable reactivity that serves as a functional synthetic equivalent of ketyl radicals through activation of the aldehyde by 
a low-valent nickel species in the presence of a silyl chloride. The 1,1-difluoroalkenes obtained through reductive couplings of aldehydes with trifluoromethyl-substituted alkenes with extrusion of a single fluorine atom are intriguing motifs owing to their presence in a number of biologically active compounds. ${ }^{[23]}$ Due to their resistance to in vivo metabolism, gem-difluoroalkenes are a promising carbonyl bioisostere owing to their steric and electronic similarity to aldehydes, ketones, and esters. ${ }^{[24]}$ Herein, we describe efficient nickelcatalyzed defluorinative couplings of trifluoromethyl-substituted alkenes with aliphatic aldehydes to provide homoallylic alcohols possessing the gem-difluoroalkenes structural motif.

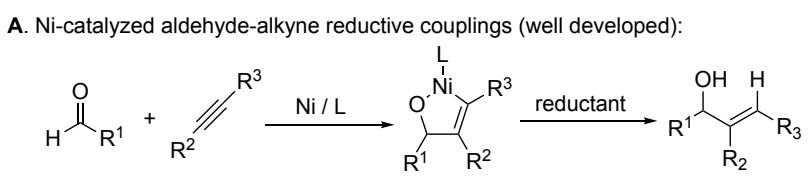

B. Ni-catalyzed aldehyde-alkene cross-couplings (more limited):

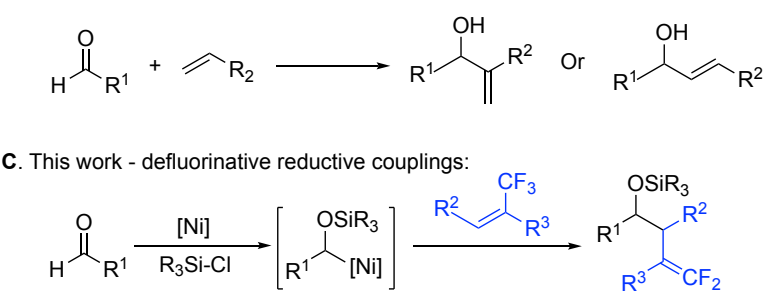

Scheme 1. Nickel-catalyzed additions to alkynes and alkenes.

Initial experiments focused on the coupling of hydrocinnamaldehyde (1a) with a trifluoromethyl alkene (2a) (Table 1). Through systematic investigation of the reaction parameters, optimal results were found using a combination of $\mathrm{NiCl}_{2}\left(\mathrm{DME}^{\mathrm{a}}\right), 6,6^{\prime}$-disubstituted bipyridine ligand $\mathbf{L 1}$, LiCl, 1,5-hexadiene, chlorotriethylsilane (TESCl), and nanopowder zinc as sacrificial reductant, providing $\mathbf{3 a}$ in $83 \%$ isolated yield (entry 1). Control experiments showed that the nickel source, ligand (L1), nanopowder zinc, and 1,5-hexadiene (entries 2-7) each played a pivotal role in this transformation. Although the inclusion of LiCl did not significantly affect yields of the standard product (entry 8), it resulted in modest improvements in yield with other substrates. Importantly, olefin additives can minimize the formation of enol ether (Scheme 1,9) and silyl ether (Scheme 1, 10) side products, and 1,5-hexadiene provided superior reactivity compared with other olefin additives (entries 9-13).[21b] It should be noted $\mathrm{Ni}(\mathrm{cod})_{2}$ had a comparable efficiency to $\mathrm{NiCl}_{2}(\mathrm{DME})$, but $\mathrm{NiCl}_{2}$ (DME) was employed due to its stability in air and ease of handling (entry 19). Finally, the ligand selection was essential for the reaction outcome, and the 2,2'-bipyridine framework provided optimal results with 6,6'-disubstitution providing further improvements, leading to L1 (6,6'-dimethyl-2,2'-bipyridine) as the optimal choice from our studies (entries 1, 14). Other nitrogen-based ligands such as Biox, Terpy, phosphines such as $\mathrm{PCy}_{3}$, or NHC ligands such as IMes led to lower yields (entries 15-18).

We next turned our attention to define the substrate scope using the optimized conditions from the above studies. First, we explored an array of aliphatic aldehydes $\mathbf{1}$ to examine the generality of the coupling with trifluoromethyl alkenes (2a) (Table 2). Unhindered aliphatic aldehydes were well tolerated, delivering the corresponding products in good yield (3c-3m). The presence of $\beta-$ substituents (3b) and a-substituents (3n-3q) were also competent participants albeit with diminished efficiency. Notably, a number of potentially reactive functional groups were unaffected in the transformation, including aryl chlorides (3c), aryl bromides (3d), aryl boronate esters (3e), and alkynes (3f). Benzyl ethers $(\mathbf{3 g})$, silyl ethers $(\mathbf{3 h})$, acetals $(\mathbf{3} \mathbf{i})$, esters $(\mathbf{3} \mathbf{j})$, and carbamates $(\mathbf{3 p})$ were also well tolerated. In addition, heterocyclic substrates including furans (3I) and indoles $(\mathbf{3 m})$ were also suitable coupling partners in the 
process. When the substrate contains both an aldehyde and ketone functional group (3k), the reaction is completely selective for aldehydes, leaving the ketone unchanged.

We next demonstrated the generality of this protocol with respect to the trifluoromethyl alkenes $\mathbf{2 a}-\mathbf{t}$ (Table 3 ). Under these mild and base-free conditions, various 1,1-trifluoromethylstyrenes featuring either electron-rich (4a-4e) or electron-deficient (4g-4k) substituents underwent the transformation smoothly, affording the corresponding products in good yields (71-93\%). Notably, this reductive protocol is tolerant of a wide range of functionality on the alkene coupling partner, such as esters (4i), amides (4j), sulfonyl groups (4k), nitriles (4I), and sulfides (4m). Furthermore, heterocycles including quinolone (4o), benzofuran (4p), benzothiophene (4q), dibenzofuran (4r), and carbazole (4s), are also readily compatible. It is noteworthy that beyond the aryl and heteroaryl system, monosubstituted alkenes, such as 2-nonafluorobutyl-1alkene (2t), smoothly proceeded to afford the desired product $4 \mathbf{t}$ in moderate yield.

Table 1. Optimization of couplings of aldehydes with trifluoromethylalkenes

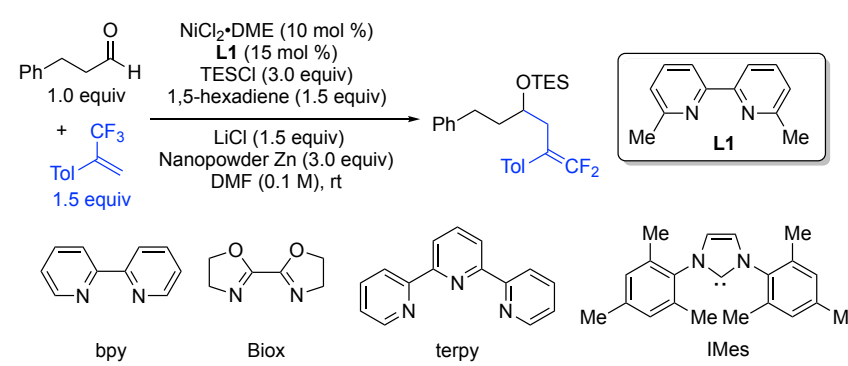

\begin{tabular}{|c|c|c|}
\hline Entry & Deviation from Standard Conditions & $\%$ Yield $^{a}$ \\
\hline 1 & None & $86(83)^{b}$ \\
\hline 2 & $\mathrm{NiCl}_{2} \cdot \mathrm{DME}$ omitted & --- \\
\hline 3 & L1 omitted & 6 \\
\hline 4 & nanopowder Zn omitted & -- \\
\hline 5 & Zinc dust ${ }^{c}$ & 67 \\
\hline 6 & Mn powder ${ }^{c}$ & 71 \\
\hline 7 & 1,5-hexadiene omitted & 37 \\
\hline 8 & LiCl omitted & 80 \\
\hline 9 & 1,5 -cyclooctadiene ${ }^{d}$ & 49 \\
\hline 10 & 1,7 -octadiene ${ }^{d}$ & 37 \\
\hline 11 & 1,6-heptadiene ${ }^{d}$ & 67 \\
\hline 12 & $(E)$-stilbene $^{d}$ & 68 \\
\hline 13 & duroquinone $^{d}$ & 6 \\
\hline 14 & bpye & 80 \\
\hline 15 & $\mathrm{BiO}^{\mathrm{e}}$ & 7 \\
\hline 16 & Terpy ${ }^{e}$ & -- \\
\hline 17 & $\mathrm{PCy}_{3}{ }^{e}$ & -- \\
\hline 18 & $\mathrm{IMes}^{\mathrm{e}}$ & 3 \\
\hline 19 & $\mathrm{Ni}(\mathrm{COD}){ }_{2}^{\mathrm{f}}$ & 86 \\
\hline
\end{tabular}

a: Yields were determined by GC with $n$-tridecane as the internal standard. $b$ : Isolated yield from a 0.2 mmol preparative experiment. $c$ : additive in place of nanopowder zinc. $d$ : additive in place of 1,5-hexadiene. e: ligand instead of $\mathbf{L} 1$. $f$ : catalyst instead of $\mathrm{NiCl}_{2} \cdot \mathrm{DME}$ 
To showcase the robustness and practicality of our method, a 5-mmol-scale experiment was conducted under an inert atmosphere using a benchtop setup without glovebox manipulations to provide $1.5 \mathrm{~g}$ of the desired product $3 \mathrm{a}$ in $73 \%$ yield using only $2 \mathrm{~mol} \%$ catalyst loading (Scheme 2A). Additionally, the protocol was also expanded to include $\beta$-trifluoromethyl enoates. As shown in Scheme 2B, subjecting methyl 4,4,4-trifluorocrotonate (5) to this catalytic system exclusively provided the defluorinative alkylation product 6 in high yield, illustrating that the trifluoromethyl group directs regiochemistry of the addition in analogy to the examples provided in Tables 2 and 3. Alkyl-substituted trifluoromethyl alkene (7), however, did not participate in the process, and competitive formation of enol ether 9 and reduced silyl ether 10 was observed, with most of trifluoromethyl alkene $\mathbf{7}$ recovered intact with only $6 \%$ yield of the desired product 8 observed by GCMS analysis.

While this manuscript was in preparation, a related chromium-catalyzed method for the addition of ketyl radicals to trifluoroalkene intermediates was described. ${ }^{[25]}$ Notably, the use of nickel catalysis was described in that study as ineffective in promoting the reaction, illustrating the unique effectiveness of the ligand/additive/reductant combination developed herein. Control experiments illustrated that $\mathrm{CrCl}_{3}$, used in the method of Wang, has little effect on rates or outcomes of our optimal nickel-catalyzed conditions, suggesting that co-catalysis with trace chromium is not involved in the method describe herein.

Table 2. Aldehyde scope in couplings with trifluoromethyl alkenes.

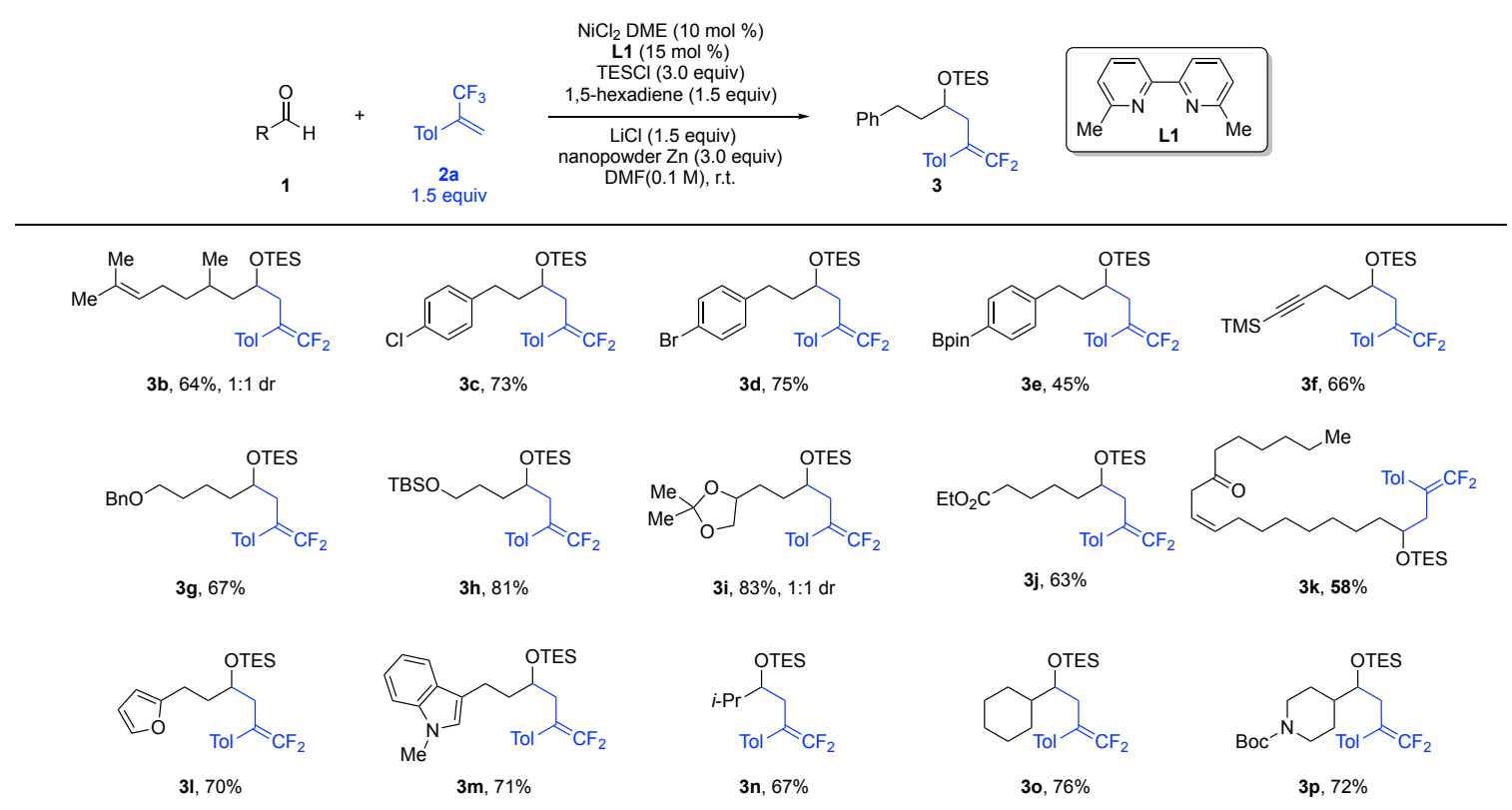

a: Reactions performed on $0.20 \mathrm{mmol}$ scale unless otherwise noted. Yields are for isolated material. Tol $=p$-tolyl. 


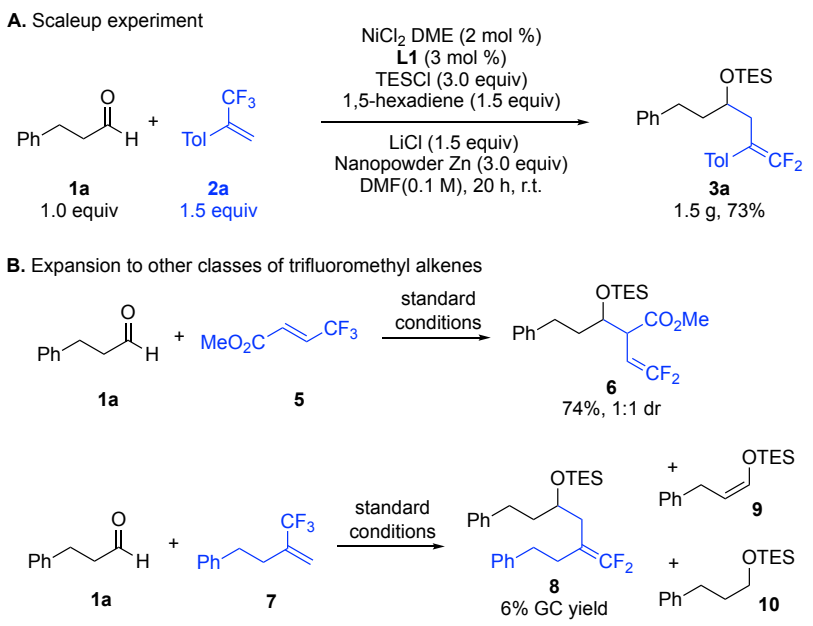

Scheme 2. Scaleup experiment and additional substrate classes.

A description of possible reaction pathways is outlined (Scheme 3). The addition of $\mathrm{Ni}(0)$, generated from the reduction of the $\mathrm{Ni}(\mathrm{II})$ pre-catalyst, to the aldehyde $1 \mathrm{a}$ in the presence of chlorotriethylsilane (TESCl) provides a possible route to $\mathrm{Ni}(\mathrm{II})$ intermediate I complexed with electron-deficient trifluoromethyl alkene (2a). Intermediate I could undergo single electron reduction with $\mathrm{Zn}$ to afford the corresponding $\mathrm{Ni}(\mathrm{I})$ intermediate II. Free radical species have previously been proposed as intermediates derived from homolytic bond scission of nickel alkyl species in other classes of nickel-catalyzed trifluoroalkene addition reactions, ${ }^{[22 \mathrm{~g}, 22 \mathrm{k}]}$ and it is plausible that free ketyl intermediate III could potentially be derived from either intermediate I or II. The observation of product 3a is consistent with either an organometallic addition of I or II to provide intermediate $\mathbf{V}$, which would undergo nickel fluoride elimination to afford product $\mathbf{3 a}$, or from addition of a transiently generated ketyl radical III.

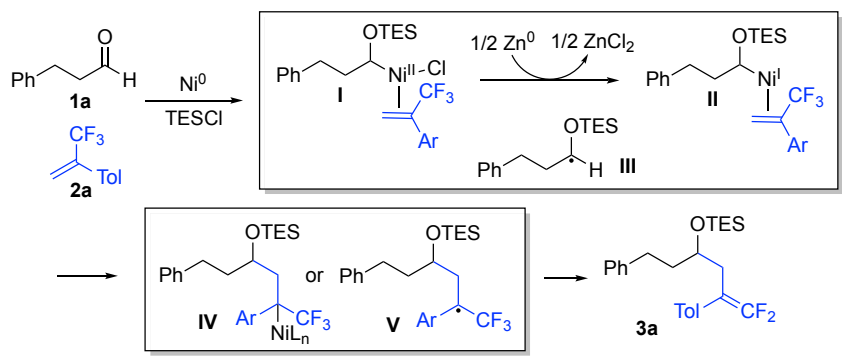

Scheme 3. Possible intermediates involved in key mechanistic steps. 
Table 3. Trifluoromethyl alkene scope in couplings with aldehydes.

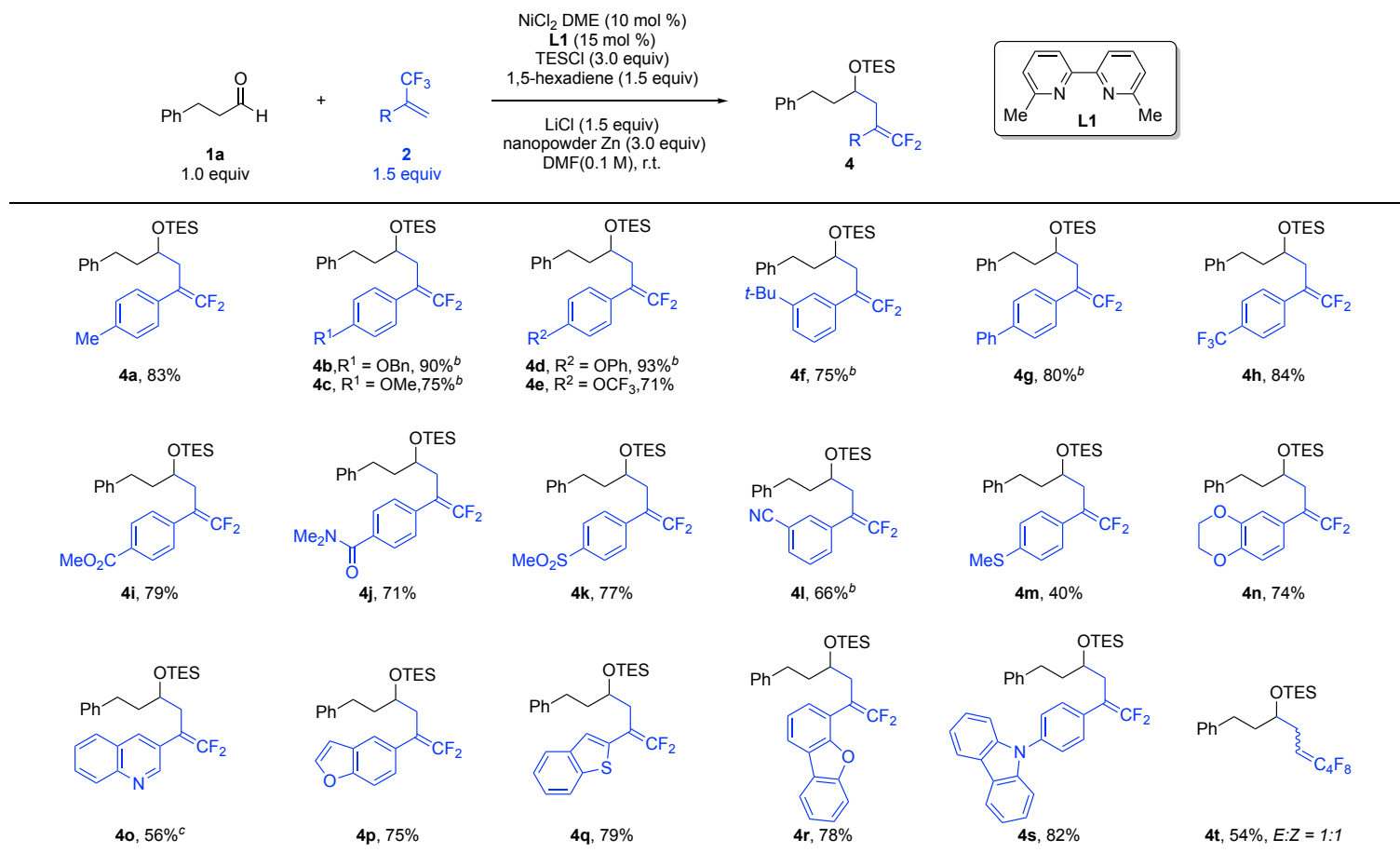

a: Reactions performed on $0.20 \mathrm{mmol}$ scale unless otherwise noted. Yields are for isolated material; $b$ : Reactions performed with $0.40 \mathrm{mmol}$ aldehyde and $0.20 \mathrm{mmol}$ trifluoromethyl alkenes; c: Reactions performed with $0.24 \mathrm{mmol}$ aldehyde and 0.20 mmol trifluoromethyl alkenes.

In conclusion, an efficient method for defluorinative cross-couplings of aliphatic aldehydes with trifluoromethyl alkenes has been developed. The facile installation of the difluoromethylene unit to an array of aldehyde structures provides an effective entry to this desirable functional group class. The substrate scope enables wide variation of the aldehyde reaction partner, and the protocol is amenable to gram-scale syntheses. The combination of a hindered 6,6'-disubstituted bipyridine ligand, 1,5-hexadiene as an additive, triethylsilyl chloride, and nanopowder zinc were key components of the optimized procedure. This work expands the use of simple alkenes in nickel-catalyzed reductive couplings of aldehydes and illustrates that bipyridine ligand frameworks enable unique reactivity in processes of this type when used in combination with simple diene additives.

\section{Acknowledgements}

The authors thank the National Institute of General Medical Sciences (R35-GM118133) for support of this research. Dr. Cole

L. Cruz is thanked for helpful suggestions. 


\section{References}

[1] a) M. J. Krische, Springer: Berlin 2007; b) D. A. Everson, D. J. Weix, J. Org. Chem. 2014, 79, 4793-4798; c) T. Moragas, A. Correa, R. Martin, Chem. Eur. J. 2014, 20, 8242-8258; d) D. J. Weix, Acc. Chem. Res. 2015, 48, 1767-1775; e) X. Wang, Y. Dai, H. Gong, Top. Curr. Chem. 2016, 374, 43.

[2] a) E. Oblinger, J. Montgomery, J. Am. Chem. Soc. 1997, 119, 9065-9066; b) X.-Q. Tang, J. Montgomery, J. Am. Chem. Soc. 2000, 122, 6950-6954; c) G. M. Mahandru, G. Liu, J. Montgomery, J. Am. Chem. Soc. 2004, 126, 3698-3699; d) H. A. Malik, G. J. Sormunen, J. Montgomery, J. Am. Chem. Soc. 2010, 132, 6304-6305; e) E. P. Jackson, H. A. Malik, G. J. Sormunen, R. D. Baxter, P. Liu, H. Wang, A. R. Shareef, J. Montgomery, Acc. Chem. Res. 2015, 48, 1736-1745; f) E. P. Jackson, J. Montgomery, J. Am. Chem. Soc. 2015, 137, 958-963; g) W. S. Huang, J. Chan, T. F. Jamison, Org. Lett. 2000, 2, 4221-4223; h) K. M. Miller, W. S. Huang, T. F. Jamison, J. Am. Chem. Soc. 2003, 125, 3442-3443; i) R. M. Moslin, K. M. Miller, T. F. Jamison, Tetrahedron 2006, 62, 7598-7610; j) E. A. Standley, S. Z. Tasker, K. L. Jensen, T. F. Jamison, Acc. Chem. Res. 2015, 48, 1503-1514; k) N. Saito, T. Katayama, Y. Sato, Org. Lett. 2008, 10, 3829-3832; I) C. Y. Zhou, S. F. Zhu, L. X. Wang, Q. L. Zhou, J. Am. Chem. Soc. 2010, 132, 10955-10957.

[3] a) R. Sawaki, Y. Sato, M. Mori, Org. Lett. 2004, 6, 1131-1133; b) Y. Sato, Y. Hinata, R. Seki, Y. Oonishi, N. Saito, Org. Lett. 2007, 9, 55975599 .

[4] a) S. K. Kang, S. K. Yoon, Chem. Commun. 2002, 2634-2635; b) J. Montgomery, M. Song, Org. Lett. 2002, 4, 4009-4011; c) S. S. Ng, T. F. Jamison, J. Am. Chem. Soc. 2005, 127, 7320-7321; d) M. Song, J. Montgomery, Tetrahedron 2005, 61, 11440-11448.

[5] a) S. Ogoshi, M. A. Oka, H. Kurosawa, J. Am. Chem. Soc. 2004, 126, 11802-11803; b) S. Ogoshi, M. Ueta, T. Arai, H. Kurosawa, J. Am. Chem. Soc. 2005, 127, 12810-12811; c) Y. Hoshimoto, Y. Hayashi, H. Suzuki, M. Ohashi, S. Ogoshi, Angew. Chem. Int. Ed. 2012, 51, 10812-10815; d) Y. Hayashi, Y. Hoshimoto, R. Kumar, M. Ohashi, S. Ogoshi, Chem. Commun. 2016, 52, 6237-6240; e) Y. Hayashi, Y. Hoshimoto, R. Kumar, M. Ohashi, S. Ogoshi, Chem. Lett. 2017, 46, 1096-1098; f) Y. Hoshimoto, Y. Hayashi, M. Ohashi, S. Ogoshi, Chem. Asian J. 2017, 12, 278-282.

[6] a) K. Ogata, Y. Atsuumi, D. Shimada, S. Fukuzawa, Angew. Chem. Int. Ed. 2011, 50, 5896-5899; b) K. Ogata, A. Toh, D. Shimada, S.-i. Fukuzawa, Chem. Lett. 2012, 41, 157-158; c) J. S. Ahlin, N. Cramer, Org. Lett. 2016, 18, 3242-3245.

[7] K. Ogata, Y. Atsuumi, S. Fukuzawa, Org. Lett. 2010, 12, 4536-4539.

[8] L.-J. Xiao, X.-N. Fu, M.-J. Zhou, J.-H. Xie, L.-X. Wang, X.-F. Xu, Q.-L. Zhou, J. Am. Chem. Soc. 2016, 138, $2957-2960$.

[9] a) S. S. Ng, T. F. Jamison, J. Am. Chem. Soc. 2005, 127, 14194-14195; b) C. Y. Ho, S. S. Ng, T. F. Jamison, J. Am. Chem. Soc. 2006, 128, 5362-5363; c) S. S. Ng, C. Y. Ho, T. F. Jamison, J. Am. Chem. Soc. 2006, 128, 11513-11528; d) C. Y. Ho, T. F. Jamison, Angew. Chem. Int. Ed. 2007, 46, 782-785; e) C. Y. Ho, K. D. Schleicher, C.-W. Chan, T. F. Jamison, Synlett 2009, 2009, $2565-2582$.

[10] J. L. M. Matos, S. Vasquez-Cespedes, J. Gu, T. Oguma, R. A. Shenvi, J. Am. Chem. Soc. 2018, $140,16976-16981$.

[11] Y. L. Zheng, Y. Y. Liu, Y. M. Wu, Y. X. Wang, Y. T. Lin, M. Ye, Angew. Chem. Int. Ed. 2016, 55, 6315-6318.

[12] X. W. Han, T. Zhang, Y. L. Zheng, W. W. Yao, J. F. Li, Y. G. Pu, M. Ye, Q. L. Zhou, Angew. Chem. Int. Ed. 2018, 57, 5068-5071.

[13] K. J. Garcia, M. M. Gilbert, D. J. Weix, J. Am. Chem. Soc. 2019, 141, 1823-1827.

[14] a) Z. Zuo, D. T. Ahneman, L. Chu, J. A. Terrett, A. G. Doyle, D. W. MacMillan, Science 2014, 345, 437-440; b) K. M. Arendt, A. G. Doyle, Angew. Chem. Int. Ed. 2015, 54, 9876-9880; c) J. L. Jeffrey, J. A. Terrett, D. W. MacMillan, Science 2015, 349, 1532-1536; d) A. Noble, S. J. McCarver, D. W. MacMillan, J. Am. Chem. Soc. 2015, 137, 624-627; e) R. Karimi-Nami, J. C. Tellis, G. A. Molander, Org. Lett. 2016, 18, 2572-2575; f) B. J. Shields, A. G. Doyle, J. Am. Chem. Soc. 2016, 138, 12719-12722; g) J. Twilton, M. Christensen, D. A. DiRocco, R. T. Ruck, I. W. Davies, D. W. C. MacMillan, Angew. Chem. Int. Ed. 2018, 57, 5369-5373.

[15] a) D. J. Hart, Science 1984, 223, 883-887; b) D. J. Edmonds, D. Johnston, D. J. Procter, Chem. Rev. 2004, 104, 3371-3404; c) K. C. Nicolaou, S. P. Ellery, J. S. Chen, Angew. Chem. Int. Ed. 2009, 48, 7140-7165; d) A. Peter, S. Agasti, O. Knowles, E. Pye, D. J. Procter, Chem. Soc. Rev. 2021, 50, 5349-5365.

[16] a) B. E. Kahn, R. D. Rieke, Chemical Reviews 1988, 88, 733-745; b) J. E. Mcmurry, Chemical Reviews 1989, 89, 1513-1524; c) E. J. Corey, G. Z. Zheng, Tetrahedron Lett. 1997, 38, 2045-2048; d) J. Streuff, Synthesis 2013, 45, 281-307; e) M. Szostak, N. J. Fazakerley, D. Parmar, D. J. Procter, Chem. Rev. 2014, 114, 5959-6039.

[17] a) K. T. Tarantino, P. Liu, R. R. Knowles, J. Am. Chem. Soc. 2013, 135, 10022-10025; b) L. J. Rono, H. G. Yayla, D. Y. Wang, M. F. Armstrong, R. R. Knowles, J. Am. Chem. Soc. 2013, 135, 17735-17738.

[18] a) M. A. Ischay, M. E. Anzovino, J. Du, T. P. Yoon, J. Am. Chem. Soc. 2008, 130, 12886-12887; b) J. Du, K. L. Skubi, D. M. Schultz, T. P. Yoon, Science 2014, 344, 392-396; c) K. N. Lee, Z. Lei, M. Y. Ngai, J. Am. Chem. Soc. 2017, 139, 5003-5006; d) C. X. Ye, Y. Y. Melcamu, H. H. Li, J. T. Cheng, T. T. Zhang, Y. P. Ruan, X. Zheng, X. Lu, P. Q. Huang, Nat. Commun. 2018, 9, 410.

[19] L. Wang, J. M. Lear, S. M. Rafferty, S. C. Fosu, D. A. Nagib, Science 2018, 362, 225-229.

[20] a) P. Hu, B. K. Peters, C. A. Malapit, J. C. Vantourout, P. Wang, J. Li, L. Mele, P. G. Echeverria, S. D. Minteer, P. S. Baran, J. Am. Chem. Soc. 2020, 142, 20979-20986; b) X. Zhang, C. Yang, H. Gao, L. Wang, L. Guo, W. Xia, Org. Lett. 2021, 23, $3472-3476$. 
[21] a) K. W. Shimkin, J. Montgomery, J. Am. Chem. Soc. 2018, 140, 7074-7078; b) C. Cruz, J. Montgomery, ChemRxiv 2021, DOI 10.26434/chemrxiv.14369888.v14369881.

[22] a) Y. Lan, F. Yang, C. Wang, ACS. Catal. 2018, 8, 9245-9251; b) Z. Lin, Y. Lan, C. Wang, ACS. Catal. 2018, 9, 775-780; c) D. Ding, Y. Lan, Z. Lin, C. Wang, Org. Lett. 2019, 21, 2723-2730; d) Y. He, D. Anand, Z. Sun, L. Zhou, Org. Lett. 2019, 21, 3769-3773; e) Z. Lin, Y. Lan, C. Wang, Org. Lett. 2019, 21, 8316-8322; f) X. Lu, X. X. Wang, T. J. Gong, J. J. Pi, S. J. He, Y. Fu, Chem. Sci. 2019, 10, 809-814; g) F. Chen, X. Xu, Y. He, G. Huang, S. Zhu, Angew. Chem. Int. Ed. 2020, 59, 5398-5402; h) H. W. Du, Y. Chen, J. Sun, Q. S. Gao, H. Wang, M. D. Zhou, Org. Lett. 2020; i) Y. Jin, J. Wu, Z. Lin, Y. Lan, C. Wang, Org. Lett. 2020, 22, 5347-5352; j) Z. Lin, Y. Lan, C. Wang, Org. Lett. 2020, 22, 3509-3514; k) C. Zhu, Z. Y. Liu, L. Tang, H. Zhang, Y. F. Zhang, P. J. Walsh, C. Feng, Nat. Commun. 2020, 11, 4860; I) W. J. Yue, C. S. Day, R. Martin, J. Am. Chem. Soc. 2021, 143, 6395-6400.

[23] a) M. Bobek, I. Kavai, E. De Clercq, J. Med. Chem. 1987, 30, 1494-1497; b) Y. Pan, J. Qiu, R. B. Silverman, J. Med. Chem. 2003, 46, 5292-5293; c) K. Muller, C. Faeh, F. Diederich, Science 2007, 317, 1881-1886; d) S. Purser, P. R. Moore, S. Swallow, V. Gouverneur, Chem. Soc. Rev. 2008, 37, 320-330; e) S. Messaoudi, B. Treguier, A. Hamze, O. Provot, J. F. Peyrat, J. R. De Losada, J. M. Liu, J. Bignon, J. Wdzieczak-Bakala, S. Thoret, J. Dubois, J. D. Brion, M. Alami, J. Med. Chem. 2009, 52, 4538-4542.

[24] a) C. Leriche, X. He, C. W. Chang, H. W. Liu, J. Am. Chem. Soc. 2003, 125, 6348-6349; b) G. Magueur, B. Crousse, M. Ourévitch, D. Bonnet-Delpon, J.-P. Bégué, Journal of Fluorine Chemistry 2006, 127, 637-642; c) N. A. Meanwell, J. Med. Chem. 2011, 54, $2529-2591$.

[25] C. Zhang, Z. Lin, Y. Zhu, C. Wang, J. Am. Chem. Soc. 2021, 143, 11602-11610. 